\title{
O EL CONTROL DEL ESPACIO ESCOLAR: CONDICIONES HIGIÉNICAS Y PEDAGÓGICAS DE LAS ESCUELAS (ESPAÑA, 1857-1931)
}

\author{
Francisco Martín Zuñiga ${ }^{1}$ \\ Maria del Carmen Sanchidrián Blanco ${ }^{2}$
}

\section{RESUMEN}

En este artículo se describe la legislación acerca de las condiciones higiénicas de escuelas, desde la Ley Moyano (1857) hasta el final de la Restauración (1931) y se interpretan a la luz de fuentes primarias -informes administrativos y escritos de maestros donde describían sus escuelas- que contrastan con las teorías pedagógicas e higienistas de la época. La principal conclusión es que los poderes municipales, los responsables, incumplieron la normativa alquilando locales frecuentemente insalubres, donde el alumnado se veía privado de elementos esenciales como luz, aire y espacio. El control sobre las condiciones pedagógicas e higiénicas era prácticamente inexistente.

Palabras clave: enseñanza primaria, políticas educativas, espacio escolar, higiene escolar.

\footnotetext{
${ }^{1}$ Universidad de Málaga (UMA), Málaga, Espanha.

2 Universidad de Málaga (UMA), Málaga, Espanha.
} 


\section{O CONTROLE DO ESPAÇO ESCOLAR: CONDIÇÕES HÍGIÉNICAS E PEDAGÓGICAS DAS ESCOLAS (ESPANHA, 1857-1931)}

\section{RESUMO}

Este artigo descreve a legislação sobre as condições de higiene das escolas, da Lei Moyano (1857) até o final da Restauração (1931) e é interpretada à luz de fontes primárias -relatórios administrativos e escritos de professores onde Eles descreveram suas escolas- que contrastam com a teoria pedagógica e higienista da época. A principal conclusão é que as autoridades municipais, as responsáveis, quebraram as regras alugando instalações com freqüência pouco saudáveis, onde os alunos ficaram privados de elementos essenciais como a luz, o ar e o espaço. $\mathrm{O}$ controle sobre as condições pedagógicas e de higiene era praticamente inexistente.

Palavras-chave: educação primária, políticas educacionais, espaço escolar, higiene escolar.

\section{THE CONTROL OF SCHOOL SPACE: HYGIENIC AND PEDAGOGICAL CONDITIONS OF SCHOOLS (SPAIN, 1857-1931)}

\section{ABTSRACT}

This article describes the legal measures regarding the hygienic conditions of school buildings from the Moyano Act (1857) until the end of the Monarchical Restoration (1931). They are interpreted in the light of primary sources - administration reports and writings of teachers describing their school buildings- that contrast with the hygienic and pedagogical theories of that time. The main conclusion is that local authorities, who were the responsible, failed to fulfill those regulations and used to rent unhealthy buildings, where children were deprived of essential elements such as light, air and space. The pedagogical and hygienic conditions were really uncontrolled.

Keywords: Primary education, Educational policies, School space, School hygiene.

\section{LE CONTRÔLE DE L'ESPACE SCOLAIRE: CONDITIONS HYGIÉNIQUES ET PÉDAGOGIQUES DES ÉCOLES (ESPAGNE, 1857-1931)}

\section{RÉSUMÉ}

Cet article décrit la législation sur les conditions d'hygiène dans les écoles, de la Loi de Moyano (1857) à la fin de la Restauration (1931) et est interprété à la lumière des sources primaires rapports administratifs et écrits des enseignants où Ils ont décrit leurs écoles - qui contrastent avec la théorie pédagogique et hygiéniste de l'époque. La conclusion principale est que les autorités municipales, les responsables, ont enfreint les règles en louant des locaux souvent malsains, où les étudiants ont été privés d'éléments essentiels tels que la lumière, l'air et l'espace. Le contrôle sur les conditions pédagogiques et hygiéniques était pratiquement 
inexistant.

Mots-clés: éducation primaire, politiques éducatives, espace scolaire, hygiène scolaire. 


\title{
INTRODUCCIÓN
}

\begin{abstract}
iLas escuelas! No hay quien sostenga que las tenemos buenas. En asambleas, en conferencias, en libros y periódicos es frecuente oír y leer que se les llama cárceles sombrías, focos de infección, antesalas de hospital, o que se las compara con sentinas de barco negrero, con estufas infectas de plantas enfermizas, cuando no con inmundas pocilgas (MARTÍ ALPERA, 1904).

El aula debe representar para el niño "lo que el gabinete de trabajo para el naturalista, el geógrafo, el historiador, el político, el literato: un sitio de reposo donde afirmar, ordenar y construir las ideas y datos recogidos en el campo, en el museo, en la fábrica, en la sociedad, en medio de la vida, en suma, a donde hay que llevar la escuela, porque es donde se aprende" (COSSÍO, 1902, p. 2-3).
\end{abstract}

Estos dos textos prácticamente coetáneos, denunciaban con crudeza la distancia existente entre la teoría y la realidad de nuestras escuelas. Sin embargo, higiene y pedagogía habían estado unidas en teoría desde que se fue configurando el sistema educativo liberal, cuando se señaló que uno de los objetivos de la escuela obligatoria era mejorar la salud de los niños. Para ello, era necesario proporcionarles unos espacios donde pudieran desarrollarse física e intelectualmente según las exigencias de la higiene y la pedagogía.

En España, los historiadores de la educación prestaron una atención tardía a los aspectos materiales de nuestra historia de la educación. No obstante, la historiografía en torno a los espacios escolares creció exponencialmente a partir del monográfico de Historia de la Educación coordinado por ANTONIO VIÑAO FRAGO, donde había interesantes aportaciones, de entre las que destacaremos las del autor mencionado (1993-1994 a y b) y la de ESTEBAN MATEO y LÓPEZ MARTÍN (1993-1994). Posteriormente, se han ido publicando diversos trabajos de una variada temática sobre las condiciones higiénicas y pedagógicas de las escuelas, lo mismo que las referidas al higienismo en general (ALCAIDE, 1999a y 1999b). En algunos casos se trata de estudios acerca de una ciudad o provincia como Sevilla (AÑóN ABAJAS, 2005), Guadalajara (ALONSO MARAÑÓN y PASTOR PRADILLO, 1993), Madrid (RODRÍGUEZ MÉNDEZ, 
2004) o Málaga (MARTÍN ZÚÑIGA, 2012), mientras que otros, como LAHOZ (1992), LÁZARO (1975), LÓPEZ MARTÍN (1997), VIÑAO FRAGO (2008) o VISEDO (1986 y 1991), afrontan la problemática de las construcciones escolares en nuestro país subrayando la relevancia que el análisis de los espacios escolares tiene para los historiadores de la educación. El hecho de que congresos como los organizados por la Sociedad para el Estudio del Patrimonio Histórico-Educativo (SEPHE) en 2016 tuviera como tema central Espacios y Patrimonio HistóricoEducativo (DÁVILA \& NAYA, 2016) y el de la ISCHE de 2019 Places and Spaces, son también indicadores del interés nacional e internacional de los historiadores de la educación por los espacios de aprendizaje.

Tanto en las primeras décadas del siglo XIX, con Montesino y Monlau, como cien años después, dentro de la Escuela Nueva, con Montessori y Decroly, los médicos jugaron un papel importante en la vida diaria de las escuelas (MILEWSKI, 2014). La inspección médica, las campañas de vacunación, las colonias escolares, las cantinas escolares, entre otras iniciativas, fueron reflejo de esa relación entre médicos y pedagogos que, salvo excepciones, existió sólo en teoría en los casi cien años considerados (PETRINA, 2006). Pero la higiene en los espacios escolares es un tema poliédrico, no sólo atañe a pedagogos y médicos, sino que también afecta a arquitectos, urbanistas, políticos o la misma Iglesia, y todos tienen su propio punto de vista (TERRÓN, 2000). Además, cada perspectiva puede verse desde la teoría, la legislación y la práctica, como todos los temas que abordamos en Historia de la educación.

Nuestra perspectiva es el estudio de las medidas de control propuestas por el poder político para mejorar las condiciones espaciales e higiénicas de los edificios destinados a escuelas públicas de enseñanza primaria, viendo su aplicación -o inaplicación- en la realidad escolar del momento. Abarcamos desde la aprobación de la Ley de Instrucción Pública de 9 de septiembre de 1857 (Ley Moyano), la primera que regula el sistema educativo español y de una vigencia más que centenaria, hasta que en 1970 se promulga la Ley General de Educación, y concluimos ante las puertas de la II República (1931). Junto a la 
legislación, describiremos las precarias condiciones higiénicas -y por tanto pedagógicas- de los espacios escolares donde los niños debían pasar buena parte de su infancia, demostrando que la corriente higienista surgida en favor de aquéllos en Europa a finales del siglo XIX, y defendida por pedagogos insignes dentro y fuera de nuestro país, apenas incidió en las políticas de mantenimiento y creación de centros públicos de enseñanza primaria.

Para hacer frente a este tema, nos hemos centrado en el examen e interpretación de fuentes primarias como textos legales, información generada por la propia administración de la enseñanza, informes de la inspección, circulares de las Juntas Provinciales de Instrucción Pública ${ }^{3}$ y toda aquella documentación donde maestros y maestras nos revelan, desde su punto de vista, el estado del espacio donde desempeñaba su función docente, tomando como muestra para esta ultima cuestión un estudio realizado sobre lo manifestado por el magisterio primario malagueño de esos años.

Pretendemos demostrar que el control ejercido por los poderes municipales sobre las condiciones higiénicas y pedagógicas de los locales destinados a escuelas fue escaso, aunque su obligación legal fuera velar por el buen estado de los mismos. Esta lamentable situación llevó a algunos ediles a hacer propuestas para mejorarlos (SANCHIDRIÁN y MARTÍN, 2013), aunque no se produjeran cambios significativos en la realidad escolar pese a los discursos higienistas y a algunas buenas intenciones legislativas.

En función de la hipótesis planteada, iniciaremos nuestro estudio reflexionando sobre los supuestos higienistas defendidos a finales del siglo XIX

\footnotetext{
3 Estas fueron creadas por la mencionada Ley de Instrucción Pública de 1857 en las capitales de provincia. Las presidía el Gobernador Civil junto a un Diputado provincia, un Consejero provincial, un representante de la Comisión Provincial de Estadística, un Catedrático de Instituto, un representante del ayuntamiento, el Inspector de Escuelas de la provincia, un eclesiástico delegado de la Diócesis y dos o más padres de familia (art. 281). Sus funciones eran la de informar al Gobierno; promover la mejora y adelantos de los establecimientos de primera y segunda enseñanza; vigilar sobre la buena administración de los fondos de los establecimientos y de las faltas que advirtieran en la enseñanza y régimen de los Institutos y escuelas puestas (art. 286). Por otro lado, también se aprobaron la Juntas Locales de Enseñanza Primaria a quienes se les encomendó la organización de dicha enseñanza a nivel municipal.
} 
y principios del XX y su repercusión en la legislación que regula la construcción de los centros de primaria en esos años, y concluiremos aterrizando en la realidad que nos muestra maestros y maestras sobre las condiciones deplorables de los locales destinados a escuelas públicas.

\section{HIGIENISMO Y ESCUELA}

El movimiento higienista-sanitario surgió en Europa a finales del siglo XIX y se introdujo en España a través de exiliados políticos, como sucedió con el médico y pedagogo Pablo Montesino (1781-1849) que tuvo que huir a Inglaterra durante la década ominosa (1823-1833). Allí quedó impresionado por el sanitary movement así como por las primeras experiencias de infant schools y tras su regresó a España quiso implantar aquellas experiencias. Para ello, creó la Sociedad para mejorar la educación del pueblo, fundó la Primera escuela de párvulos en 1838 y publicó su Manual para maestros de la escuela de Párvulos (1840). También colaboró en el Reglamento de las Escuelas Públicas de Instrucción Primaria Elemental de 1838, en cuyo preámbulo se establecen las bases, desde un punto de vista legal, "del discurso que inicia en nuestro país el movimiento higienista en sus relaciones con la educación. Un texto en el que, desde una perspectiva reformista, la escuela y el maestro aparecen como un instrumento privilegiado de mejora higiénica, moral y social" (VIÑAO FRAGO, 2000, pp. 10-11).

Una concepción al uso de higiene en esos momentos pudo ser la que nos ofrecieron Avendaño y Carderera en su Curso elemental de pedagogía:

Los fluidos que nos rodean, las sustancias en contacto con nuestro cuerpo, los alimentos y bebidas, el sueño y vigilia [...] ejercen una influencia saludable o nociva en la salud, según las circunstancias que acompañan su acción. La buena salud depende por tanto de precaverse de la influencia dañosa y aprovecharse de la favorable, y esto es lo que enseña la higiene, dándonos las causas que producen las 
En síntesis, se plantea una definición que ofrece, por un lado, una actuación preventiva, dando información y formación sobre todo aquello que pueda provocar la enfermedad a través, por ejemplo, de la inclusión en el currículo de una asignatura relacionada con esa temática y, por otro, se promueve la acción directa para evitar que se propague la enfermedad favoreciendo el ejercicio físico, la atención médica y unas condiciones saludables en los edificios escolares.

El movimiento higienista alcanzó un importante desarrollo en el último tercio de siglo XIX y primero del XX, impulsado por el afán modernizador del regeneracionismo ${ }^{4}$ (LAHOZ ABAD, 1992, p. 90) que, en esta cuestión, "además de consolidar una nueva imagen de la infancia, se empeñó en la búsqueda de fórmulas consecuentes para proporcionar los cuidados y la atención que aquella requería” (RUIZ RODRIGO y PALACIO LIS, 1999, p. 12).

La higiene infantil empezó a ser un tema de interés para pedagogos y maestros y prueba de ello fueron publicaciones como las de Díez Canseco (1863); Tolosa Latour (1880); Chillida Meliá (1882); Iraolagoitia (1882) y Sánchez de Castro (1882). Además, en 1883 inicia su singladura la revista La Madre y el Niño. Revista de Higiene y Educación, dirigida por Manuel Tolosa Latour y en los años siguientes van apareciendo obras como las de Bouza Dávila (1884); Alcántara García (1886); Tolosa Latour (1893) y la conocida Paidología de Domingo Barnés (1921). Además, se traducen al castellano obras de autores de relevancia internacional como El siglo de los niños Ellen Key o El alma del niño de Preyer.

\footnotetext{
4 Movimiento ideológico surgido en España a finales del siglo XIX como consecuencia de la pérdida de las colonias (Cuba, Puerto Rico y las Islas Filipinas) en 1898 y la degeneración del propio estado. Su propósito era el de regenerar el país mediante la propuesta de reformas educativas, sociales, políticas y económicas, considerando la educación como el pilar fundamental para consolidar dichas reformas. Encabezó dicho movimiento el político, jurista, economista e historiador Joaquín Costa, junto con Lucas Mallada, Ricardo Macías Picavea y Rafael Altamira, entre otros.
} 
Otras manifestaciones de esta inquietud fueron la creación en 1881 de la Sociedad Española de Higiene; la celebración en 1882 del primer Congreso Nacional de Higiene; diversas Instrucciones técnico-higiénicas aprobadas por el Ministerio de Instrucción Pública para la construcción de escuelas, de las cuales hablaremos en el próximo apartado; el Real Decreto de 26 de octubre de 1901 donde se introducen los "ejercicios corporales" en el currículum oficial de la enseñanza primaria; la aprobación en 1904 de la Ley de Protección a la Infancia; la creación de la Inspección Médica Escolar (1911) y la celebración del I Congreso Nacional de Higiene Escolar (1912), entre otros acontecimientos relevantes (VIÑAO, 2000, pp. 19-24). Sin embargo, las relaciones entre médicos y maestros en la escuela son un tema aún inexplorado en nuestro contexto, aunque otros casos pueden hacernos pensar que eran relaciones muy escasas y vistas por los maestros como una supuesta intromisión (HIRST, 1991).

Podemos concluir, que el higienismo pedagógico se reflejó en las políticas educativas desde distintas perspectivas: vinculado a la mejora de la salud de los escolares desde la propia acción educativa, con temas relacionados con educación física, colonias, ambientalismo ...; la higiene como asignatura dentro del curriculum para informar y formar hábitos de conducta saludables; la inspección médica escolar; adecuación del mobiliario a las leyes del crecimiento y las edades para prevenir y corregir deformaciones y, finalmente, las condiciones arquitectónicas de edificios, aulas, patios y demás dependencias donde se impartían las enseñanzas.

Pero fue en esta última cuestión donde el movimiento higienista mostró especial sensibilidad:

Las condiciones higiénicas exigidas para la construcción de escuelas impusieron los emplazamientos, la orientación del edificio, las dimensiones de las aulas, el número de ventanas y las dimensiones de las mismas, la dirección de la luz, los sistemas de calefacción, los servicios sanitarios, los espacios libres, los materiales de construcción, etc., influyendo sobre la conformación del edificio-escolar en general (LAHOZ ABAD, 1992, p. 90). 
Todas estas condiciones especiales dieron a la arquitectura escolar un carácter propio y una dimensión uniformadora de los espacios que, por desgracia, en el periodo que investigamos (1857-1931) no tuvo suficiente repercusión en las políticas municipales de construcción de escuelas públicas. Es cierto que el debate a inicios del siglo XX en favor de la graduación de la enseñanza primaria despertó el interés de algunos ayuntamientos (Cartagena, Barcelona, Madrid, Málaga...) por construir grupos escolares, pero siguieron predominando las escuelas unitarias, con un solo maestro al frente de un grupo de alumnos con diferentes grados académicos, ubicadas en espacios construidos con otro fin, casi siempre casas de vecinos en las que el cabildo alquilaba una vivienda a particulares. Por consiguiente, no eran espacios adecuados para la enseñanza y a menudo, como veremos en los siguientes apartados, carecían de las condiciones higiénicas de luz y ventilación necesarias.

\section{MARCO LEGISLATIVO SOBRE LAS CONDICIONES HIGIÉNICAS Y PEDAGÓGICAS DE LOS LOCALES DESTINADOS A ESCUELAS DE PRIMARIA PÚBLICAS}

Desde que empezó a diseñarse el sistema educativo a comienzos del XIX, encontramos ya con alguna normativa relacionada con esta cuestión, por ejemplo el Plan y Reglamento de escuelas de primeras letras del Reino de 16 de febrero de 1825 y el Reglamento de Escuelas Públicas de Instrucción Primaria de 26 de noviembre de 1838 , que en su art. $3^{\circ}$ especificaba que "en todos los pueblos se establecerá la escuela en lugar conveniente, que no esté destinado a otro servicio público; en sala o pieza proporcionada al número de niños que vaya a contener, con bastante luz, ventilación y defensa de la intemperie”.

La Ley de Instrucción Pública de 9 de septiembre de 1857 ratificó en el art. 97 lo establecido en el Reglamento anterior, es decir, que las escuelas públicas "estarán a cargo de los respectivos pueblos, que incluirán en sus 
presupuestos municipales, como gasto obligatorio, la cantidad necesaria para atender a ellas", aunque "todos los años, sin embargo, se consignará en el presupuesto general del Estado la cantidad de un millón de reales, por lo menos, para auxiliar a los pueblos que no puedan costear por sí solos los gastos de la primera enseñanza”. En conjunto la normativa era poco clara, lo mismo que los escritos que advirtieron de la necesidad de considerar esos aspectos (CARDERERA, 1855, pp. 253-261 y pp. 281-283; GINER DE LOS RÍOS, 1911), pues no se decía nada de cómo debían ser las escuelas y se les seguía encomendando a los ayuntamientos una responsabilidad que en la mayoría de los casos no podían asumir porque los responsables no tenían la conciencia necesaria o porque la miseria económica que reinaba en la mayoría de los municipios no les permitía destinar la partida necesaria para construir escuelas.

Las construcciones escolares específicas fueron escasas. Normalmente lo que existieron fueron los denominados locales de escuela, consistentes en habitaciones o casas alquiladas a particulares por los ayuntamientos o estancias de procedencia diversa, como cuarteles en desuso, calabozos, viejas dependencias municipales... (VISEDO,1991, p. 128). Se reducían a un único espacio ocupado por un maestro o una maestra que impartía docencia a un grupo numeroso de alumnado, por ejemplo, en Málaga lo normal era que cada docente tuviera más de cien alumnos, bastante heterogéneo en cuanto a edades y nivel de conocimientos.

Un primer paso para la estandarización y homogenización de la arquitectura escolar fue el decreto-ley de 18 de enero de 1869, donde se especificó que, además de las aulas, las escuelas debían de contar con habitación del profesor, biblioteca y jardín; también se limitaba el número de alumnos por clase a 120 y se especificaba que el volumen de las aulas debía de ser de tres metros cúbicos por alumno como mínimo dependiendo la superficie necesaria del método aplicado: $75 \mathrm{dm}^{2}$ por alumno en el caso del "simultaneo" y un $\mathrm{m}^{2} \mathrm{si}$ se aplicaba el "mutuo". Sin embargo, como indica Viñao, "la grave situación heredada, en lo que a los edificios escolares se refiere, no experimentó alivio o 
mejora alguna (...) Lo que duele, sin embargo, no es la constatación de este hecho, sino el incumplimiento por el liberalismo radical y progresista de sus promesas" (1993-1994c, p. 503).

La normativa se infringió sistemáticamente, llegando a tener que intervenir el Ministerio de Instrucción Pública y Bellas Artes ${ }^{5}$ con la publicación de sucesivas circulares, como una de 1909, donde exigía a los ayuntamientos que "realicen inmediatamente las obras necesarias para poner las escuelas públicas en las condiciones debidas o trasladarlas a locales mejores”. Un claro ejemplo de esa desidia la encontramos en la ciudad de Málaga, donde la propia Junta Provincial de Instrucción Pública manifestaba en 1903 que:

\begin{abstract}
Se hallan instaladas las Escuelas en edificios que carecen por completo de condiciones higiénicas y pedagógicas y a los cuales asisten los niños para verse privados de los elementos más esenciales y principalísimos de su vida: luz, aire, espacio, alegría. Y en una atmósfera viciada, y con un ambiente más propio de cárcel o de calabozo que de Escuela o templo del saber, el trabajo repugna, la enseñanza mortifica, las horas que se dedican a la educación resultan eternas e insoportables (BALLESTEROS Y MÁRQUEZ, 1903, p. 29).
\end{abstract}

A comienzos del XX se legislaron medidas para mejorar el estado de las escuelas públicas, coincidiendo con la creación del Negociado de Arquitectura (R.D. de 26 de septiembre de 1904), dependiente del Ministerio de Instrucción Pública, la llegada de la enseñanza graduada y las denuncias de políticos, médicos, pedagogos y maestros de la lamentable situación de estos centros. En ese sentido, tenemos que citar las Instrucciones técnico-higiénicas para la construcción de escuelas de 1905, las Notas elaboradas por el Museo Pedagógico Nacional en 1911 y la creación de la Oficina Técnica para la Construcción de Escuelas en 1920. Pero la primera normativa legal más

\footnotetext{
${ }^{5}$ La aparición de este Ministerio en la España es tardía con respecto a otros países. Se crea en el año 1900, a partir de la Ley de Presupuestos del Estado de 31 de marzo y el real decreto de 8 de abril -con anterioridad el tema educativo era un anexo de ministerios como el de Gracia y Justicia o Fomento-. Con él se inicia un proceso de extensión y fortalecimiento organizativo de la enseñanza.
} 
detallada con relación a la construcción de edificios destinados a escuelas públicas fue la especificada en el decreto de 26 de septiembre de 1904 y en las mencionadas instrucciones de 28 de abril de 1905, donde se limita a 60 el número de alumnos por clase y se contemplan mejoras en las condiciones de salubridad de los edificios destinados a escuelas:

\begin{abstract}
Cada alumno debe disponer como mínimo de un metro cuadrado dentro del aula; la extensión en conjunto de todos los huecos de ventilación e iluminación -puertas, ventanas...- tiene que ser equivalente a la cuarta parte del total; toda escuela es preciso que cuente por lo menos con un retrete y un urinario, así como con el agua necesaria para la higiene y necesidades de los alumnos (REAL DECRETO DE 28 de abril de 1905, p. 407) .
\end{abstract}

Pero, como veremos después, dicha normativa se siguió incumpliendo sistemáticamente. El problema de muchos municipios era un alto endeudamiento que rozaba la bancarrota. Por ese motivo, salvo raras excepciones, los ayuntamientos carecían de edificios construidos ex profeso para uso escolar. Las autoridades municipales preferían soportar los gastos de alquileres antes que hacer un mayor desembolso para la construcción de escuelas. Pero lo malo de esta dependencia de locales privados era que a veces primaba más el "amiguismo" que las condiciones higiénicas y pedagógicas de dichos locales. Ante esta situación, el estado opta finalmente por intervenir directamente en la construcción de las escuelas públicas a partir del R.D. de 23 de noviembre de 1920, terminando así con el monopolio municipal en esta cuestión. En el preámbulo del citado decreto se reconoce que "la intervención de los ayuntamientos no ha sido todo lo positiva que podía esperarse" y, de ahí, la mala situación de los locales destinados a escuelas. Por ello, en art. $1^{0}$ establece por fin que "La construcción de edificios escolares, tanto los destinados a escuelas graduadas, como unitarias, se realizarán por el Estado, sujetándose a los medios ordinarios de subasta o contrata, prevenidos por la Ley de Contabilidad y Administración de la Hacienda Pública de $1^{\circ}$ de junio de 1911". De todos modos, los ayuntamientos continuaron manteniendo competencias en 
la cooperación con las administraciones educativas en la creación, construcción y mantenimiento de los centros docentes públicos, siendo esta una obligación que aún se mantiene vigente en nuestros días, como se refleja en la Ley de bases del Régimen Local de 1985 (MOLINA MARTÍN, 2007, p. 47).

\section{LOS LOCALES DE LAS ESCUELAS PÚBLICAS DE MÁLAGA VISTOS POR EL PROPIO PROFESORADO $Y$ LAS AUTORIDADES RESPONSABLES DE LA ENSEÑANZA}

Analizaremos en este punto la situación real de las escuelas en la provincia de Málaga ${ }^{6}$ que en 1880 era similar a la media nacional, según los datos recogidos por la Dirección General de Instrucción Pública (1883), ya que los porcentajes de escuelas clasificadas como buenas, regulares o malas son parecidas. Sin embargo, el aspecto donde no sigue la media es en la propiedad de las escuelas ya que en España el 59,2 \% de los locales eran de propiedad municipal y en Málaga ese porcentaje es sólo del 17,75 \%. Esto obligaba a pagar alquileres con una economía que estaba en bancarrota. La situación de la capital era especialmente negativa porque las necesidades de escuelas eran mayores y porque en los ayuntamientos pequeños las posibilidades de que los ayuntamientos tuvieran algún espacio que pudiera dedicarse a escuela eran mayores, sin entrar, claro está, a discutir si eran los más adecuados o no.

Cuadro 1: Estado de los locales de las escuelas públicas: España y Málaga, 1880 Propios Alquilados

Total

\begin{tabular}{|c|c|c|c|c|c|c|c|}
\hline & Estado & N & \% & N & \% & N & \% \\
\hline & Bien & 3517 & 27,05 & 1416 & 15.51 & 4933 & 22,09 \\
\hline
\end{tabular}

\footnotetext{
${ }^{6}$ Málaga es una de las ocho provincias que constituyen lo que hoy se denomina la Comunidad Autónoma de Andalucía. Según datos del Instituto Nacional de Estadística en 1900 la provincia contaba con una población de 520385 habitantes, siendo la escolar de 57800 .
} 


\begin{tabular}{|c|c|c|c|c|c|c|c|}
\hline España & Regular & 6544 & 49,57 & 4721 & 51,72 & 11265 & 50,46 \\
\hline & Mal & 3139 & 23,38 & 2990 & 32,77 & 6129 & 27,45 \\
\hline & Total & 13200 & 59,12 & 9127 & 40,88 & 22327 & 100,00 \\
\hline \multirow{2}{*}{ Málaga } & Bien & 20 & 38,46 & 37 & 15,35 & 57 & 19,45 \\
\hline & Mal & 10 & 19,23 & 71 & 29,46 & 81 & 27,65 \\
\hline & Total & 52 & 17,75 & 241 & 82,25 & 293 & 100,00 \\
\hline
\end{tabular}

Fuente: Elaborado a partir de los datos de la Dirección General de Instrucción Pública (1883).

A finales del XIX, las escuelas de la capital y la provincia vivían en un continuo peregrinar de local en local sin que los sucesivos cambios supusieran mejoras efectivas. No era raro que, apenas trasladada una escuela, el maestro se dirigiera a la correspondiente junta local pidiendo reparaciones en el local de la misma (SANCHIDRIÁN, 1986, p. 263).

Por ese motivo, la propia Junta Provincial de Instrucción Pública de Málaga informaba en 1903 que el problema de los edificios escolares era importante, pero difícil de resolver ya que estos "influyen en la buena marcha de la enseñanza” y de ellos “depende la conveniente organización pedagógica”. La síntesis que se presenta es esta:

Edificios de pésima construcción: locales, cuando no ruinosos, viejos, destartalados y pequeños; salones de clase reducidos, de irregulares formas, unas veces estrechos, otros oscuros, otros faltos de ventilación, siempre sin condiciones pedagógicos; casas de vecindad en cualquiera de cuyos pisos se establece la escuela (BALLESTEROS Y MÁRQUEZ, 1903, p. 30).

La situación era tan desastrosa que tuvo que intervenir en el asunto el Subsecretario de Instrucción Pública, quien envió un telegrama (fechado el 7 de 
agosto de 1909) al presidente de la Junta provincial malagueña, Rosendo F. Baldor, solicitando imperiosamente que "sin excusa, ni pretexto, ni dilación exija a los Ayuntamientos realicen inmediatamente obras necesarias para poner Escuelas públicas en condiciones debidas o trasladar locales mejores”.

La Junta Provincial, ante tan abrumadoras recomendaciones, ordenó que todos los ayuntamientos durante el mes de agosto realizaran las mejoras necesarias para que las escuelas tuvieran las condiciones necesarias, ajustándose a lo establecido en el R.D. de 28 de abril de 1905 que, recordemos, especificaba que la superficie necesaria para cada alumno, la superficie total de los huecos de ventilación e iluminación y los requisitos higiénicos.

Para cumplir con estas medidas, se requería una remodelación importante de los locales -correr tabiques, agrandar o abrir nuevas ventanas-, obras que no siempre eran factibles por las dimensiones reducidas y por la estructura misma de los locales (una casa donde se han eliminado tabiques interiores y que cuenta con una ventana en un extremo), con lo cual, la única solución era el traslado de las escuelas a otros locales más apropiados o construir edificios "ex profeso" para centros escolares. De todas formas, y pese a la urgencia de las medidas anteriores, José Sanmartín, nuevo presidente de la mencionada Junta Provincial, reconoce un año más tarde que "con los dedos de la mano pueden contarse los Ayuntamientos que han reformado e higienizado sus locales de escuelas" (Circular de la Junta Provincial, 1910).

Uno de los pocos municipios que pareció preocuparse algo más por esta cuestión, aunque tarde y cuando la situación era casi insostenible, fue el de Málaga capital. Ya en 1902, durante el pleno de 14 de marzo, el concejal Sánchez Pastor solicitó que en el orden del día del próximo cabildo se incluyeran dos informes, uno del secretario de la Junta local y otro del inspector médico municipal, sobre el estado actual de las escuelas:

El primero manifestó que: 
Una lijera [sic] visita de inspección realizada a los locales que ocupan las Escuelas públicas, demuestra al más profano, hasta qué punto se han tenido en olvido aquellos preceptos [higiénicos y pedagógicos]; pues observase en unos la falta de capacidad en los solares de clase (...); en otros la falta de luz impide sean aprovechables todas las horas laborables (...); en los más, lo irregular de los locales destinados, a clases, hace ineficaz la necesaria vigilancia de los profesores (...) y por último, hay Escuelas situadas en calles estrechas (...), donde no es posible evitar que al oído de las niñas, lleguen las inmundas respiraciones del vicio...” (INFORME DEL SECRETARIO, 1902) ${ }^{7}$.

Y el inspector médico, por su parte, fue más drástico en sus afirmaciones:

Pudiera en rigor sintetizarse este informe diciendo que en Málaga no existen realmente instalaciones que merezcan dicho nombre [escuelas públicas]; que los Centros de enseñanza carecen de toda condición higiénica, y que al adquirir estos locales parece que todo se tuvo en cuenta menos los elevados fines a que estaban destinados.

Ambos informes, especialmente el segundo, muestran que el grave problema de las escuelas públicas no era su falta de condiciones pedagógicas, sino que constituían un "grave atentado" para la salud del niño -falta ventilación, luz, espacio...-, y por ello recomendaban la construcción de edificios destinados a escuelas.

El deterioro de los locales donde se situaban las escuelas es un tema que siguió apareciendo con cierta frecuencia en los plenos del ayuntamiento de Málaga; por ejemplo, el arquitecto municipal informaba en 1904 acerca del mal estado de las escuelas Nuestra Sra. del Carmen (clausurada), Nuestra Señora de la Encarnación, San Ciriaco y Santa Paula, San Antonio (clausurada), Santa Rosa, San Andrés (clausurada) y Purísima Concepción.

El 14 de septiembre de 1906, el concejal F. Ruiz Gutiérrez presentó otra

\footnotetext{
7 Todos los textos de los testimonios que se citan se encuentran en los legajos 1.768 y 1.771 del Archivo Municipal de Málaga (A.M.MA.).
} 
moción criticando la situación de los locales y proponiendo la construcción de grupos escolares como medio para corregir esa deficiencia, pero eso no haría hasta el 4 de abril de 1913, cuando los concejales Adolfo Pérez Gascón (Liberal) y Diego Martín Rodríguez (republicano), presentaron una moción en el consistorio malagueño proponiendo que "se proceda, sin pérdida de tiempo, a la formación del proyecto de un edificio destinado a Escuela Pública, en los terrenos que posee el Ayuntamiento denominados 'Huerto de la Tiña" (Actas de Sesiones, t. 311, 4-IV-1913), dándose los primeros pasos para la construcción del que sería el primer grupo escolar malagueño y andaluz, el Bergamín, inaugurado en 1916 (MARTÍN ZÚÑIGA, 2012, p. 99).

Las críticas al mal estado de las escuelas públicas tenían cierto eco social en la prensa local. Concretamente, El Popular publicó en sus páginas unas declaraciones del Delegado Regio de Instrucción Pública de Málaga, Narciso Díaz Escovar, afirmando, entre otras cosas, que "los edificios destinados a la enseñanza son, por lo regular, pequeños, de malas condiciones y sin ninguno de esos requisitos que, la pedagogía moderna señala" (DÍAZ ESCOVAR, 1915, p. 8).

Pero quienes mejor describían el estado lamentable de muchas de las edificaciones destinadas a escuelas eran los propios maestros, cuyos testimonios hemos extraído a partir de las comunicaciones enviadas a alcaldes, Delegado Regio o inspección y conservadas en el Archivo Municipal de Málaga. Por ejemplo, el maestro de la escuela de niños de Santa Ana comentaba en 1888:

\footnotetext{
A consecuencia sin duda del excesivo número de niños que concurren a esta Escuela y que con sus marchas imprimen un movimiento fuerte de trepidación al edificio, ya viejo y resentido en demasía, se ha vuelto a reproducir las grietas abiertas en sus muros por los terremotos del año 84; y como quiera que vayan aumentando de día en día en profundidad y anchura hasta llegar hoy a presentar caracteres alarmantes.
}

El arquitecto municipal coincidía con el diagnóstico del maestro y puntualizaba que el local tiene los muros agrietados y "el piso presenta cierta 
flexión que hace temer que las cabezas de las maderas no se encuentran en buen estado".

Otro maestro, el de la escuela de El Salvador, informaba en 1889 a la Junta Local de Enseñanza Primaria de Málaga que:

\begin{abstract}
De continuar las clases instaladas por mucho tiempo en estos salones que manan humedad y que expelen salistre [sic.] por suelo y paredes, en los cuales se siente un ruido escandaloso y se percibe un olor fétido insoportable, y entre los que se halla situado, contra toda prescripción higiénica, el corral que sirve de lavadero a los vecinos y de escusado a los niños se colocará en un peligro continuo e inminente de salud de los discípulos y maestros, y se dificultará lejos de favorecer el desarrollo paulatino de la organización infantil, a la cual se aminora con padecimientos de fatales consecuencias.
\end{abstract}

De esta misma escuela, años más tarde (1912), se denuncia que "carece de agua hace mucho tiempo, y el techo de la clase ofrece peligro eminente de derrumbarse (...). Los retretes están atascados por la falta de agua, resultando malos olores".

La maestra de San Juan Nepomuceno (1910) describía el local donde está instalada esta escuela de niñas como:

Un cúmulo de defectos, siendo imposible desarrollar ninguno de los sistemas de enseñanza. Su orientación y luz son viciosas y enfermizas, siendo muchas veces necesario suprimir la clase de labores tan importante a la mujer.

Encontrándose el lugar escusado próximo a la clase, siendo éste un pequeño hueco de escalera sin respiradero ni ventilación [...], los gases en descomposición que exala [sic.] aquel foco [...], vician la atmósfera en términos que las niñas y aun las mismas profesoras se sienten, a veces, molestadas por náuseas y fuertes dolores de cabeza.

Esta misma maestra también se quejaba de la inmoralidad del vecindario, pues adosada a la escuela existía una casa de "mal vivir, en donde con frecuencia se suceden escenas escandalosas e inmorales, de que las niñas se 
aperciven [sic], no obstante las muchas precauciones de la maestra".

El maestro de Nuestra Señora de los Dolores manifestaba en abril de 1902 "que la techumbre del Salón de clase (...) se encentra en eminente estado de ruina". La maestra

De la Santísima Trinidad, refiriéndose a unas obras realizadas en la escuela en 1908, comentaba:

Dichas obras están sin terminar, toda vez que las paredes y techos recompuestos se hallan en el más lamentable estado de limpieza, que dicho señor propietario se niega en absoluto a encalarlos. Así mismo se niega resueltamente a realizar la compostura y pintura de las puertas y ventanas, como la colocación de cristales.

En agosto de 1909, la maestra de la escuela de San José advertía que:

Debiendo abrirse el día de mañana las clases (...) y habiéndose verificado obras de reparaciones y sin haber terminado éstas (...) falta de muchos cristales, blanqueo y pintura, careciendo de agua todas las dependencias como así mismo el retrete que se halla instalado dentro de la clase, produciéndose un malestar y un perjuicio de la salud.

El maestro de Nuestra Señora de las Mercedes se lamentaba en septiembre de 1910:

En primer lugar, considerando muy antihigiénico el uso del jarro y la orza que usan los niños para beber, propongo se instale un surtidor (...). También creo de necesidad reformar el pavimento de madera que se halla muy deteriorado hasta el punto que raro es el día que no se registra alguna caída (...). Por último, que no teniendo la casa más puerta de entrada y salida que la puerta del salón de clase, y observando que continuamente se distrae la atención de los niños por las personas que a todas horas entran y salen, juzgo conveniente se haga construir otra entrada. 
La maestra de San Joaquín comunicaba en mayo de 1913 que, "después de tres meses sin agua, había contratado, de su propio peculio, los servicios de un "aguador", pero lo verdaderamente dramático era la descripción que hacía de las pésimas condiciones higiénicas y de las vejaciones que sufrían sus alumnas y ella misma:

\begin{abstract}
En la calle donde caen las ventanas de mi clase se ha formado, con las últimas lluvias, una laguna cuyas aguas verdes despiden un olor insoportable y son focos de infección [...] dicha calle conocida en el barrio con el nombre del Compás y que tiene su entrada por el Camino de Suárez y la Calzada de la Trinidad, está completamente abandonada, nunca la barren ni pasa por ella un guardia, por esto, está convertida en un estercolero, cuyas pestilencias respiran mis alumnas, y es campo de juego de niños sin respeto de ninguna clase que se entretienen en apedrear la escuela, cayendo muchas piedras en el patio; arrojan dentro de la clase barro, excrementos, depositan animales muertos en las ventanas, escupen y molestan con palabras soeces.
\end{abstract}

En definitiva, los comentarios anteriores nos reflejan las duras condiciones en que se desarrollaba la enseñanza en algunas escuelas públicas, con condiciones que atentaban contra la salud, la vida y la propia dignidad del alumnado y profesorado. Las quejas, como acabamos de comentar, eran muchas y diversas, y eso que sólo hemos presentado una muestra de la capital. En el resto de la provincia la situación no era mucho mejor, y prueba de ello la encontramos en las descripciones que nos dejó Luis Bello en su obra Viaje por las Escuelas de España (BELLO, 1927, pp. 93-161) de las escuelas de algunos pueblos como Alora, Casarabonela, Churriana, Fuengirola, Ronda, Torremolino.

Los culpables de esta situación no eran sólo los propietarios de los locales, sino los dirigentes municipales que, en general, se interesaban poco por la realidad de las escuelas. Esto explica que en 1880 el estado del $80 \%$ de las escuelas públicas malagueñas fuera regular o malo (cuadro 1), llegándose, incluso, a clausurar muchas de ellas por el peligro que suponía para sus moradores: por ejemplo, en el Censo escolar de 1903 se especificaba que había 
veinticuatro escuelas cerradas a nivel provincial y cinco en la capital.

Este "mal estado" incrementó el desprestigio de la enseñanza pública, favoreció aún más el desinterés del alumnado por la asistencia a clase -¿cómo obligar a los niños a asistir a una escuela que era perjudicial para su salud?-, y provocó una pérdida importante de horas lectivas mientras se efectuaban las reparaciones de los locales o se buscaban otros que normalmente también había que acondicionar (en muchos casos para mejorar poco con relación al anterior), pudiendo pasar meses o años antes de reanudarse las clases .

Llegado este punto, se puede deducir que con esas condiciones higiénicas no debió ser fácil para los maestros y maestras conseguir un rendimiento escolar adecuado de su alumnado. No podemos esperar "grandes resultados" de una enseñanza que se desarrollaba en las "pésimas condiciones" descritas, máxime cuando el propio inspector provincial proponía en 1902:

\footnotetext{
Que bajo ninguna causa y menos pretexto, se consienta que los niños permanezcan en las escuelas más que las horas reglamentarias (...) pues es perjudicialísimo para la salud de la niñez y su desarrollo físico, la estancia en la escuela durante las 3 ó 4 horas del mediodía, dadas las malas condiciones higiénicas de los locales, y por carecer estos de jardines o patios para el esparcimiento y recreo de los niños.
}

Finalmente, esta desvergüenza comenzó a hacer mella en ciertos sectores de la clase política. En ese sentido, en 1904 varios concejales socialistas y republicanos del ayuntamiento de Málaga presentaron una moción donde calificaban de delictivo el estado de las escuelas, tanto del punto de vista higiénico como pedagógico:

Las escuelas Públicas de esta Capital no reúnen las condiciones necesarias para poder realizar su cometido, sin que en las mismas se haya tenido en cuenta cómo deben los niños recibir la luz y la clase de ésta, motivo bastante para perjudicar en alto grado la vista, (...) sin que se haya tenido presente tampoco en dichos locales la altura del techo y las dimensiones oportunas del salón para que la palabra del 
maestro llegue clara a los niños, que aparte de lo que esto perjudica su cultura, determina aberraciones en la audición y torpeza consiguiente en el oído, (...) siendo el pavimento de ladrillo producen gran cantidad de polvo que hace difícil la respiración y puede acarrear graves enfermedades a los alumnos; faltando en muchas escuelas, el agua, lavabos urinarios y retretes, encontrándose los que existen de éstos en tan malas y pésimas condiciones, y tan mal colocados que sus emanaciones se perciben en la escuela, contribuyendo esto a corromper el aire y perjudicar la salud de los alumnos y profesores cometiéndose con ello un verdadero delito de lesa humanidad.

Hemos considerado importante incluir entera esta larga cita porque corresponde a los primeros años del llamado "siglo del niño", un siglo que vio profundos cambios cuantitativos y cualitativos en las escuelas desde todos los puntos de vista, aunque algunos se obstinen en negarlo. Los nuevos conocimientos sobre los niños y la educación aportados por la pedagogía, la psicología y la medicina condujeron a nuevos métodos pedagógicos y nuevas teorías acerca de lo que debía ser la escuela. Todos ellos reivindicaban la importancia de los espacios donde tienen lugar los aprendizajes.

Las nuevas corrientes arquitectónicas, especialmente el racionalismo y el funcionalismo, prestarán atención a los edificios educativos a partir de una teoría de la arquitectura aplicada a la educación. Louis Kahn, por ejemplo, uno de los mejores arquitectos de la historia, consideraba "la escuela como un ambiente espacial en el que aprender es bonito" (NORBERG-SCHULTZ. \& DIGERUD, 1981, p. 65). Faltaban décadas para que los espacios escolares de las escuelas públicas pudieran definirse así. El mal de fondo era depender económicamente de unos ayuntamientos escasos de recursos y que no consideraban que la enseñanza primaria fuera algo prioritario. Algunos municipios, como el de Málaga, incrementaron notablemente los presupuestos en instrucción pública en el primer tercio del siglo XX, pero partiendo de una situación de abandono que hacía que esos incrementos no fueran suficientes. En los Libros de Presupuestos y Ordenanzas se puede ver que de las 80.000 pesetas aprobadas para 1904 (el 2,24 \% del presupuesto) se pasó a 405.850,55 en 1924 (5,4 \%). Eso explica que las 22 escuelas unitarias (11 de niñas y 11 de 
niños) existentes en 1902 se duplicaran en 1914, a raíz de una moción presentada por la minoría republicana (8 de octubre de 1909) proponiendo el "desdoble" de aquéllas con la conversión de las "auxiliarías" en independientes, se creara el mencionado grupo escolar Bergamín (1916) y que en 1922 el número de escuelas fuera de 115 (Actas Capitulares de 10 de noviembre de 1922).

A pesar de esto, la educación primaria seguía sin ser asumida como una prioridad y, si había que hacer recortes, las partidas destinadas a educación eran las primeras en sufrirlos, como lo describe Luis Bello cuando comentaba que en los presupuestos del Ayuntamiento de Málaga para 1926-1927:

\begin{abstract}
Algún ligero sobrecargo de gastos: un campo de golf; un aumento en los gastos de representación del alcalde y del Ayuntamiento. Alguna economía. Por ejemplo: veinticinco mil pesetas por supresión de gratificaciones a médicos inspectores de escuelas; veintisiete mil pesetas por la supresión del comedor escolar de la zona de Levante; diez mil pesetas por la supresión de la subvención para sostenimiento del Instituto de Niños Anormales (BELLO, 1927, p. 148-149).
\end{abstract}

Vistas las partidas donde se hicieron recortes, es evidente que, a pesar de declaraciones donde se afirmaba que la enseñanza primaria pública era la base de la sociedad, el fundamento de la regeneración social, etc., cualquier motivo era bueno para dedicarle menos dinero. Tampoco se terminó con los problemas higiénicos y pedagógicos de las escuelas públicas, ni se crearon las necesarias para cubrir las necesidades: baste señalar que según el Anuario Estadístico de 1930 en la provincia de Málaga faltaban 700 escuelas.

Finalmente, merece la pena señalar que las condiciones de la mayoría de las escuelas privadas eran similares a las mencionadas de las públicas. Con relación a esto, habría que distinguir entre los grandes colegios privados, por ejemplo, el colegio San Estanislao de los jesuitas, donde había alumnos que cursaban allí la enseñanza primaria, pero su principal foco era el bachillerato, y contaban con edificios construidos ad hoc, y las pequeñas escuelas primarias distribuidas por la ciudad y situadas, como las escuelas públicas, en locales de 
casas particulares. Éstas eran tan deficientes, desde el punto de vista higiénico, como las públicas y ni cumplían con los requisitos exigidos ni se planteaban exigencias en las cuestiones pedagógicas (materiales de enseñanza, métodos, distribución de las clases, organización, etc.) (MARTín ZÚÑIGA, 2016, p. 479498).

\section{A MODO DE EPÍLOGO}

En el presente trabajo hemos analizamos las medidas propuestas por el poder político para mejorar las condiciones espaciales e higiénicas de los locales reservados a escuelas públicas unitarias de enseñanza primaria, desde la Ley Moyano (1857) hasta el final de la Restauración (1931), y su repercusión en la realidad escolar malagueña de ese momento.

De entrada, ha quedado demostrada la hipótesis inicial del escaso control ejercido por los poderes municipales sobre las condiciones higiénicas y pedagógicas de estos establecimientos, aunque hay que reconocer que esta lamentable situación golpeó en la conciencia de algunos ediles, especialmente en la capital, y ello les llevó a promover, a lo largo del primer tercio del siglo XX, mociones que culminaron con la creación de más escuelas, iniciaron la graduación o la construcción de grupos escolares.

Por boca del propio magisterio hemos conocido el mal estado de unas escuelas ubicadas, generalmente, en habitaciones de una casa particular de reducidas dimensiones, con falta de higiene, luz, ventilación y, en ocasiones, con un entorno vecinal inapropiado, dejando entrever las duras condiciones en las que desempeñaba su tarea docente. En definitiva, una situación en total sintonía con aquellas palabras que recordábamos al principio de Martí Alpera (1904) que calificaba a las escuelas como "cárceles sombrías, focos de infección, antesalas de hospital”,

No se puede achacar esta desidia a la falta de una normativa legal 
reguladora, pues las propuestas higienistas de finales del XIX hicieron bastante mella en las políticas educativas, como lo demuestra la abundancia de textos legales publicados en el primer tercio del XX y que comentamos en este trabajo. Sucedía, simplemente, que la normativa se infringía sistemáticamente y que de poco servían las interpelaciones del propio Ministro de Instrucción Pública exigiendo a los ayuntamientos que "realicen inmediatamente las obras necesarias para poner las escuelas públicas en las condiciones debidas o trasladarlas a locales mejores".

Esa exigencia se transformaba en súplica en las diversas comunicaciones que enviaban maestros y maestras a los alcaldes solicitando la reparación de unos locales cuyas condiciones atentaban frecuentemente contra la salud, la vida, e incluso la propia dignidad del alumnado y profesorado. Por desgracia, la respuesta de los poderes municipales fue, con frecuencia, el desprecio, la inoperancia o la incompetencia para resolver la problemática; es decir, hacían dejación de sus competencias en la creación, construcción y mantenimiento de los centros docentes públicos a las que les obligaba la ley. A pesar de esto, hemos de admitir que el movimiento higienista colaboró en el proceso de modernización de la escuela primaria desarrollado en las primeras décadas del siglo XX en nuestro país (MORENO, 2006), aunque las fuertes limitaciones de fondo y la inestabilidad política limitaron el alcance de ese desarrollo.

Finalmente, a lo anterior hay que añadir el alto endeudamiento de muchos ayuntamientos, a los que se les exigía asumir determinadas competencias sin contar con los medios económicos precisos y sin estar convencidos de la necesidad social de impulsar la educación pública. Por estos motivos, los municipios, en general, carecían de edificios construidos ex profeso para uso escolar y preferían soportar los gastos de alquileres antes que hacer un mayor desembolso para la construcción de escuelas. Una vez más estamos ante un tema en el que el mal de raíz es el incumplimiento de la normativa existente, característica de un estado débil donde las pugnas ideológicas consumieron la 
energía que podría haberse destinado a mejorar la realidad.

\section{REFERENCIAS}

ALCAIDE GONZÁLEZ, Rafael. Las publicaciones sobre higienismo en España durante el período 1736-1939. Un estudio bibliométrico. Scripta Nova. Revista electrónica de Geografía y Ciencias Sociales, vol. III, n. 37, 1999 a.

ALCAIDE GONZÁLEZ, Rafael. La introducción y el desarrollo del higienismo en España durante el siglo XIX. Precursores, continuadores y marco legal de un proyecto científico y social. Scripta Nova. Revista Electrónica de Geografía y Ciencias Sociales, n. 5O, 1999b.

ALCÁNTARA GARCÍA, Pedro de. Tratado de higiene escolar: Guía teóricopráctica. Madrid: Librería de Hernando, 1886.

ALONSO MARAÑÓN, Pedro Manuel Y PASTOR PRADILLO, José Luis. Higiene escolar y educación física en las escuelas primarias de Guadalajara a finales del siglo XIX. Wad-al-Hayara: Revista de Estudios de Guadalajara, n. 20, p, 241252, 1993 .

AÑÓN ABAJAS, Rosa María. La arquitectura de las escuelas primarias municipales de Sevilla hasta 1937. Sevilla: Universidad de Sevilla, 2005.

AVENDAÑO, Joaquín y CARDERERA, Mariano. Curso elemental de pedagogía. $5^{\text {a }}$ ed. Madrid: Imprenta de D. Victoriano Hernando, 1861.

BALLESTEROS Y MÁRQUEZ, Salvador. Memoria sobre el estado actual y las reformas más convenientes de la enseñanza primaria en esta provincia que en cumplimiento del Real Decreto de 2 de Septiembre de 1902 remite la Junta provincial de Instrucción Pública de Málaga a la Subsecretaría del Ramo. Aprobada en la sesión del 14 de Marzo de 1903. Alcalá de Henares, Archivo General de la Administración, leg. 6212, 1903 .

BARNÉS, Domingo. Paidología. Bilbao: Espasa Calpe, 1932.

BOUZA DAVILA, Ramón. La higiene de la escuela. Santiago: Imp. Cervantes, 1904.

BELLO, Luis. Viaje por las Escuelas de España. Por Andalucía: Cádiz, 
Málaga y Granada; Las dos Castillas: Toledo y Soria. Madrid: Magisterio Español, T. II, 1927.

CARDERERA, Mariano. Diccionario de educación y métodos de enseñanza. Madrid: Imp. de A. Vicente, T. II, 1885.

CHILLIDA MELIÁ, Julio. Higiene de las escuelas. Castellón: La Asociación Tipográfica, 1882.

COSSÍO, Manuel Bartolomé. Notas sobre construcción escolar. Madrid: R. Rojas, 1902.

DÁVILA, Paulí y NAYA Luis Mari. (Coord.). Espacios y patrimonio histórico-educativo. San Sebastián: Universidad del País Vasco-Erein, 2016.

DÍAZ ESCOVAR, Narciso. Instrucción Pública. El Popular, T. 4, 1915.

DÍEZ CANSECO, Vicente. (1863) Catecismo higiénico para los niños, $2^{a}$ ed. León: Establecimiento Tipográfico de Miñón, 1863.

DIRECCIÓN GENERAL DE INSTRUCCIÓN PÚBLICA. Estadística general de $1^{\text {a }}$ enseñanza correspondiente al decenio que terminó en 31-XII1880. Madrid: Imp. Manuel Tello, 1883.

ESTEBAN MATEO, León y LÓPEZ MARTÍN, Ramón. Escuela y espacio: testimonios y textos. Historia de la educación: Revista Interuniversitaria, n. 12-13, p. 75-96, 1993-1994.

GINER DE LOS RÍOS, Francisco. Notas sobre construcción escolar. Madrid: Museo Pedagógico Nacional, 1911.

HIRST, J. David (1991) Public health and the public elementary schools, 1870-1907. History of Education, n. 20:2, p. 107-118.

IRAOLAGOITIA, Antonio de (1882) Higiene escolar. Bilbao: Imprenta del Boletín Oficial, 1882.

LAHOZ ABAD, Purificación. Higiene y arquitectura escolar en la España contemporánea (1838-1936). Revista de Educación, n. 298, p. 89-118, 1992. http://laescueladelarepublica.es/wpcontent/uploads/2016/11/higiene_y_arquitectura_escolar.pdf

LÁZARO FLORES, Emilio. Historia de las construcciones escolares en España. Revista de Educación, n. 240, p. 114-126, 1975.

http://www.mecd.gob.es/dctm/revista-de-educacion/1975240/1975re240estudios11.pdf?documentId=0901e72b818204fe 
LÓPEZ MARTÍN, Ramón. La construcción y creación de escuelas en la España del primer tercio del siglo XX. Historia de la educación: Revista Interuniversitaria, n. 16, p. 65-90, 1997.

MARTÍ ALPERA, Félix. Por las Escuelas de Europa. Madrid: Sucesores de Hernando, 1904.

MARTÍN ZÚÑIGA, Francisco. La enseñanza primaria en Málaga durante el reinado de Alfonso XIII. Málaga: Diputación Provincial de Málaga, 1993.

MARTÍN ZÚÑIGA, Francisco. Los inicios de la escuela graduada en Málaga (1913-1931): El Grupo Escolar "Bergamín”. Moreno, Pedro Luís y Sebastián Vicente, Ana (eds.). Patrimonio y Etnografía de la escuela en España y Portugal durante el siglo XX. Murcia: SPHE, CEME, p. 99-111, 2012.

MARTÍN ZÚÑIGA, Francisco. La inspección de centros privados en el primer tercio del Siglo XX y el espacio escolar: el caso malagueño, en Dávila Balsera, Paulí y Naya Garmenci, Luis María. Espacios y patrimonio Históricoeducativo. Donostia, Erein Argitaletxea, p. 479-498, 2016.

MILEWSKI, Patrice. Medico-science and school hygiene: a contribution to a history of the senses in schooling. Paedagogica Historica, n. 50:3, p. 285300, 2014.

MOLINA MARTÍN, Susana. La ciudad como agente educador: condiciones para su desarrollo. Estudios sobre Educación, n. 13, p. 39-56, 2007.

MORENO MARTÍNEZ, Pedro Luis. The Hygienist Movement and the Modernization of Education in Spain. Paedagogica Historica, n. 42:6, p. 793-815, 2006. DOI: 10.1080/00309230600929542

NORBERG-SCHULTZ, Christian y DIGERUD, J.G. Louis Kahn. Idea e imagen.Madrid, 1981. Xarait.

http://www.albertoburgos.es/profile/texts/kahn_txt.htm

PETRINA, Stephen. The Medicalization of Education: A Historiographic Synthesis. History of Education Quaterly, n. 46-4, p. 503-531, 2006.

RODRÍGUEZ MÉNDEZ, Francisco Javier. Arquitectura escolar en España: 1857-1936: Madrid como paradigma. Tesis doctoral, E.T.S. Arquitectura, Universidad Politécnica de Madrid, 2004. http://oa.upm.es/254/ RUIZ RODRIGO, Cándido y PALACIO LIS, Irene. Higienismo, educación ambiental y previsión escolar. Valencia: Universidad de Valencia, 1999. 
SÁNCHEZ DE CASTRO, Lesmes. Higiene doméstica: Cartilla higiénica para uso de las escuelas. León: Imp. de los Herederos de Miñón, 1882.

\section{SANCHIDRIÁN BLANCO, Carmen. Política educativa y enseñanza} primaria en Málaga durante la Restauración (1874-1902). Málaga: Universidad de Málaga, 1986.

SANCHIDRIÁN BLANCO, Carmen y MARTÍN ZÚÑIGA, Francisco. El paso de escuelas unitarias a escuelas graduadas: Planos de escuelas en Málaga (18791916), en Espigado Tozino, Gloria, Gómez Fernandez, Juan, De la Pascua Sánchez , María José, Sánchez Villanueva, Juan Luis y Vázquez Domínguez, Carmen (eds.). La Constitución de Cádiz. Genealogía y desarrollo del sistema educativo liberal. Cádiz: UCA, p. 677-688, 2013.http://dialnet.unirioja.es/servlet/articulo?codigo $=4515028$

TERRÓN BAÑUELOS, Aída. La higiene escolar: un campo de conocimiento disputado. Áreas: Revista Internacional de ciencias sociales, n. 20, p. 7394, 2000.

TOLOSA LATOUR, Manuel. El niño. Apuntes científicos. Madrid: Imp. de la Gaceta Universal, 1880.

TOLOSA LATOUR, Manuel. (1893) Medicina e higiene de los niños.Madrid: Administración de la Revista de Medicina y Cirugía prácticas, 1893.

VALLE, Genaro del. La Higiene para las escuelas de primera enseñanza. Madrid: C. González, 1860.

VIÑAO FRAGO, Antonio. El espacio escolar: Introducción. Historia de la educación: Revista Interuniversitaria, n. 12-13, p. 11-16, 1993-1994a.

VIÑAO FRAGO, Antonio. Del espacio escolar y la escuela como lugar: propuestas y cuestiones. Historia de la educación: Revista Interuniversitaria, n. 12-13, p. 17-74, 1993-1994b.

VIÑAO FRAGO, Antonio. Higiene, salud y educación en su perspectiva histórica. Áreas: Revista internacional de ciencias sociales, n. 20, p. 924, 2000.

VIÑAO FRAGO, Antonio. Construcciones y edificios escolares durante el Sexenio Democrático (1868-1874). Historia de la Educación, Revista Interuniversitaria, n. 12-13, p. 493-534, (1993-1994c).

VIÑAO FRAGO, Antonio. Escolarización, edificios y espacios escolares. CEE Participación Educativa, n. 7, p. 16-27, 2008. 
VISEDO GODÍNEZ, José Miguel. La construcción escolar primaria en los centros públicos españoles de 1857 a 1985: Evolución histórica y análisis comparativo. Tesis doctoral. Universidad de Murcia, 1986. http://www.tesisenred.net/handle/10803/10770?show

VISEDO GODÍNEZ, José Miguel. Espacio escolar y reforma de la enseñanza. Revista Interuniversitaria de Formación del Profesorado, n. 11, p. 125135, 1991.

VISEDO GODÍNEZ, José Miguel. Legislación comentada sobre espacios y construcciones escolares de los centros públicos primarios en España: de 1825 a 1991. Anales de Pedagogía, n. 11, p. 229-244, 1993.

FRANCISCO MARTIN ZUÑIGA es profesor Titular de Historia de la Educación en la España contemporânea. Departamento de Teoría e Historia de la Educación de la Educacion y Métodos de Investigación en Educación de la Univesrsidad de Málaga (España).

E-mail: fmartinz@uma.es

(i) http://orcid.org/0000-0002-9803-8845

MARIA DEL CARMEN SANCHÍDRIAN BLANCO es catedrática de Historia de la Educación. Departamento de Teoría e Historia de la Educación de la Educacion y Métodos de Investigación en Educación de la Univesrsidad de Málaga (España)

E-mail: sanchidrian@uma.es

(b) http://orcid.org/0000-0003-4208-2182

Recebido em: 10 de dezembro de 2018

Aprovado em: 04 de junho de 2019

Revista História da Educação - RHE

Associação Sul-Rio-Grandense de Pesquisadores em História da Educação - Asphe

Artigo de acesso aberto distribuído nos termos de licença Creative Commons. 